\title{
ALUMBRADO PÚBLICO URBANO FOTOVOLTAICO: ANÁLISIS DE VIABILIDAD TÉCNICA, ECONÓMICA Y SOCIAL
}

\author{
Rami D. Orejón-Sanchez \\ Alfonso Gago-Calderón \\ Dpto. Expresión Gráfica, Diseño y Proyectos \\ Universidad de Málaga
}

Artículo Recibido: 28/04/2018

Artículo Aceptado: 14/09/2018

\begin{abstract}
Resumen
Este trabajo analiza la viabilidad de proyectos consistentes en instalaciones de alumbrado público (AP) alimentadas con energía solar fotovoltaica en base a diferentes criterios. En primer lugar se plantea un análisis de la capacidad tecnológica existente en este mercado para alumbrado exterior. En segundo lugar se comparan los requisitos, trabajos necesarios, problemática y costes identificables entre las opciones de planificar y ejecutar el soterramiento del tendido eléctrico y la instalación de nuevas luminarias fotovoltaicas (PV) LED autónomas para cubrir los requisitos normativos exigidos de alumbrado público de calles y vías. Este punto se plantea en base al marco de un caso práctico localizado en un municipio de la Isla de Lanzarote, Las Palmas, con una población en torno a 5.000 habitantes.

El trabajo se completa presentando un estudio estadístico que valora la aceptación social de ciudadanos españoles asociada a este tipo de tecnología en entornos urbanos, desde los aspectos de la mejora de la sostenibilidad urbana, la seguridad de funcionamiento, el impacto visual y la percepción del riesgo de vandalismo.
\end{abstract}

\section{Abstract}

This research analyses, based on different criteria, the viability of projects consisting of public street lighting installations powered by photovoltaic solar energy. First, an analysis of the existing technological capacity in this market for photovoltaics (PV) street lighting is generated. Later, the requirements, work, problems and costs 


\section{WPSReview International on Sustainable \\ Housing and Urban Renewal (RI-SHUR)}

identified are studied for two possible options to renew an aerial grid public lighting installation: channelling the electrical wiring or installing new autonomous LED PV luminaires to cover the regulatory requirements. This comparison is contextualized in the context of a study case located in a municipality with a population of approximately 5,000 habitants in the Island of Lanzarote (Las Palmas, Spain)

The paper is completed presenting a statistical study that assesses the social acceptance of Spanish citizens of this type of technology in urban environments, from the aspects of improving urban sustainability, operational safety, visual impact and perception of risk of vandalism.

\section{Palabras clave}

Alumbrado público, iluminación fotovoltaica, proyectos sostenibles, Green cities

\section{Key words}

Public lighting, photovoltaic lighting, sustainable projects, Green cities

\section{1.- Introducción}

A partir del desarrollo de bombillas incandescentes a finales del siglo XIX, se han investigado diversos métodos y tecnologías, tanto para producir luz blanca de manera eficiente como para generar y distribuir la energía para su funcionamiento. La tecnología basada en diodos emisores de luz (LED) supone el último gran hito en este sector tecnológico en virtud de su mayor ahorro energético, fiabilidad y fácil control electrónico con respecto al resto de tecnologías implantadas en el AP. La naturaleza digital de estos nuevos emisores tiene además como característica relevante su modo de alimentación, directamente en corriente continua (DC de ínglés direct current) y muy baja tensión (Gago A, 2011).

En la actualidad 1.300 millones de personas, el 20\% de la población mundial, no tienen acceso a una red de distribución de electricidad en su vivienda. Esto supone un problema global que se puede equiparar a la limitación de acceso a la alimentación, al agua potable o la sanidad (Carrasco-Moreno LM, 2015). En la década de 1960 se desarrollaron las primeras luminarias solares fotovoltaicas para solventar los requerimientos de iluminación en lugares sin conexión a la red eléctrica. Estos equipos se han ido desarrollando comercialmente hasta nuestros días utilizando principalmente como fuente de luz lámparas compactas fluorescentes (CFL) (Ramchandra, 2011).

En la actualidad, la generalización de las luminarias LED ha supuesto una nueva revolución tecnológica dentro de este segmento de productos. El aumento de los costes de la electricidad y de los combustibles fósiles (Nowak S, 2015), junto con el descenso exponencial en el precio del Watio-pico (Wp) generado con paneles fotovoltaicos (ARE, 2015) y el aumento consistente de la eficiencia de los emisores LED, ha propiciado que las instalaciones de iluminación LED PV se posicionen como una opción tecnológicamente eficiente y económicamente viable para cubrir las 


\section{WPSReview International on Sustainable \\ Housing and Urban Renewal (RI-SHUR)}

necesidades del alumbrado público de las ciudades; no solo para proyectos en los que son la única opción viable sino también para la renovación del alumbrado público urbano, donde las luminarias con alimentación en corriente alterna no tenían competencia hasta el momento (Hyder, 2018). Este punto tiene especial relevancia en zonas con instalaciones eléctricas muy envejecidas o deterioradas, de manera que se evita sustituirlas o adaptarlas a la normativa vigente ahorrando partidas de costes muy significativas como la de su canalización subterránea (Battaglini, 2012).

\section{2.- Estado del arte}

\section{1.- Iluminación}

En la actualidad, el $20 \%$ de la generación eléctrica del planeta se dedica a la iluminación, un 17\% a iluminación interior y un 3\% a iluminación exterior. En cualquiera de los dos casos cualquier pequeña mejora supone un importante avance en sostenibilidad y en ahorro económico (Montoya, 2017). En Europa, se estima que las ciudades y pueblos destinan, en promedio, el $40 \%$ de su presupuesto municipal en cubrir el gasto energético asociado a la iluminación de sus calles y edificios públicos (Lau SP, 2014).

En España, el consumo en iluminación exterior en instalaciones de titularidad pública o privada cuyo flujo luminoso se proyecta sobre un espacio abierto de uso público, en 2012 fue de 5,2 TWh (IDAE, 2017). Es más, el consumo de energía en alumbrado público crece en torno a un 2,6\% anual, lo que constituye un incremento en el gasto de las arcas de las Administraciones Públicas en un 4,7\%. En algunos municipios, el consumo de energía en alumbrado público alcanza hasta el $80 \%$ del consumo total de electricidad (Gutiérrez-Escolar A, 2015).

\section{2.- Tecnología de iluminación LED}

La tecnología LED ha destacado sobre el resto de dispositivos de iluminación, principalmente por su naturaleza electrónica. Esta naturaleza le ha permitido alcanzar altos valores de eficiencia energética y de vida útil, superando los $300 \mathrm{Im} / \mathrm{W}$ en pruebas de laboratorio y las 100.000 horas de vida, respectivamente (Montoya FG, 2017). A continuación, en la tabla 1, aparece un pequeño resumen comparativo de las eficiencias y vidas útiles de las diversas tecnologías de iluminación existentes. 


\section{WPSReview International on Sustainable}

\section{Housing and Urban Renewal (RI-SHUR)}

Tabla 1. Resumen de tiempos de vida y eficiencias promedias de las tecnologías de iluminación

\begin{tabular}{|c|c|c|}
\hline Sistema de alumbrado & Vida útil & Rendimiento \\
\hline \multicolumn{3}{|c|}{ Incandescencia } \\
\hline Convencional & $1.000 \mathrm{~h}$ & $12-18 \mathrm{Im} / \mathrm{W}$ \\
\hline Halógena & $2.000 \mathrm{~h}$ & $18-22 \mathrm{~lm} / \mathrm{W}$ \\
\hline \multicolumn{3}{|c|}{ Lámpara de descarga } \\
\hline $\begin{array}{l}\text { Vapor de mercurio a baja presión } \\
\text { (VMBP) }\end{array}$ & $\begin{array}{c}5.000-15.000 \\
h\end{array}$ & $38-91 \mathrm{Im} / \mathrm{W}$ \\
\hline $\begin{array}{l}\text { Vapor de mercurio a alta presión } \\
\text { (VMAP) }\end{array}$ & $8.000 \mathrm{~h}$ & $40-60 \mathrm{Im} / \mathrm{W}$ \\
\hline VMAP - Luz mezcla & $6.000 \mathrm{~h}$ & $20-60 \mathrm{Im} / \mathrm{W}$ \\
\hline VMAP - Halogenuros metálicos (HM) & $9.000 \mathrm{~h}$ & $60-95 \mathrm{Im} / \mathrm{W}$ \\
\hline Vapor de sodio a baja presión (VSBP) & $6.000-8.000 \mathrm{~h}$ & $160-180 \mathrm{~lm} / \mathrm{W}$ \\
\hline Vapor de sodio a alta presión (VSAP) & $\begin{array}{c}8.000-12.000 \\
h\end{array}$ & $130 \mathrm{~lm} / \mathrm{W}$ \\
\hline Lámpara fluorescente compacta & $8.000 \mathrm{~h}$ & $60 \mathrm{Im} / \mathrm{W}$ \\
\hline \multicolumn{3}{|c|}{ Plasma } \\
\hline Plasma & $30.000 \mathrm{~h}$ & $85 \mathrm{Im} / \mathrm{W}$ \\
\hline \multicolumn{3}{|c|}{ Iluminación en estado sólido } \\
\hline LEDs & $>100.000 \mathrm{~h}$ & $>300 \mathrm{~lm} / \mathrm{W}$ \\
\hline
\end{tabular}

Fuente: Elaboración propia

\section{3.- Generadores de energía fotovoltaica}

La célula solar es el elemento fundamental de cualquier módulo fotovoltaico, en ella se producen todos los fenómenos de conversión de energía solar a energía eléctrica. Las células están creadas con materiales semiconductores dopados con otros materiales. Por su parte, el efecto fotovoltaico es el resultado de convertir un fotón en energía eléctrica.

La energía solar fotovoltaica es una energía renovable, inagotable, no contaminante y que contribuye al desarrollo sostenible. Debido al aumento de producción de paneles fotovoltaicos en China, el coste de los mismos ha disminuido. Esto favorece a la tecnología y al uso de esta energía, puesto que se sitúa a unos precios competitivos con respecto a otras energías renovables. El crecimiento de potencia instalada de energía fotovoltaica mundial se sitúa entre el 20- 25\% cada año (Breyer, 2017).

\section{4.- Tecnologías de almacenamiento de energía: Baterías}

Las baterías están formadas por electrolitos que se encargan de almacenar la energía a partir de procesos químicos. Estos electrolitos pueden estar formados por distintos elementos. Los más comunes son el $\mathrm{Pb}$-ácido, el $\mathrm{Ni}-\mathrm{Cd}$ y, actualmente el más 


\section{WPSReview International on Sustainable \\ Housing and Urban Renewal (RI-SHUR)}

eficiente, el ion-litio. El uso y desarrollo de baterías representa uno de los elementos básicos para un cambio de modelo energético. Son elementos imprescindibles para el desarrollo de sectores estratégicos como los vehículos eléctricos o para asentar definitivamente a las placas fotovoltaicas y al resto de fuentes renovables como segmento principal del mix energético (Gils, 2017).

De esta manera, la Agencia Internacional de la Energía (AIE o IEA, del inglés International Energy Agency) señala que desde el año 2008 el coste de las baterías se ha dividido por cuatro, mientras que la densidad para almacenar energía de las mismas se ha multiplicado por cinco (IEA, 2017).

\section{3.- Requerimientos normativas para iluminación urbana}

La "Guía Técnica de Aplicación: Eficiencia Energética en Instalaciones de Alumbrado Exterior" generada por el Gobierno de España (Ministerio de Industria, Energía y Turismo, 2013) desarrolla los criterios de la International Commission on Illumination (CIE) o la Illuminating Engineering Society (IES) para establecer una base normativa de referencia, en donde se establecen las clasificaciones de tipos de vía en función de sus requisitos lumínicos.

El nivel de iluminación requerido por cada tipo de vía depende de múltiples factores como son la complejidad de su trazado, la intensidad, velocidad máxima y los sistemas de control del tráfico o la separación entre carriles destinados a distintos tipos de usuarios. En función de estos criterios, estas vías de circulación se clasifican en varios grupos o situaciones de proyecto, asignándose a cada uno de ellos, unos requisitos fotométricos específicos, que tienen en cuenta las necesidades visuales de los usuarios, así como aspectos medioambientales. Uno de los criterios más importantes a la hora de clasificar las vías, es la velocidad de circulación para cada tipo de vía, que queda establecida en la tabla 2.

Tabla 2. Clasificación de las vías según la velocidad de circulación

\begin{tabular}{|c|c|c|}
\hline Clasificación & Tipo de vía & $\begin{array}{c}\text { Velocidad del tráfico rodado } \\
(\mathbf{k m} / \mathbf{h})\end{array}$ \\
\hline A & de alta velocidad & $\mathrm{v}>60$ \\
\hline B & de moderada velocidad & $30<\mathrm{v} \leq 60$ \\
\hline C & carriles bici & -- \\
\hline D & de baja velocidad & $5<\mathrm{v} \leq 30$ \\
\hline E & vías peatonales & $\mathrm{v} \leq 5$ \\
\hline
\end{tabular}

Fuente: Ministerio de Industria, Energía y Turismo del Gobierno de España (2013)

WPS RI-SHUR, nำ-8, 2018, vol.1, ISSN: 2387-1768 


\section{WPSReview International on Sustainable \\ Housing and Urban Renewal (RI-SHUR)}

\section{4.-Análisis de viabilidad de la solución fotovoltaica urbana}

\section{1.- Análisis de viabilidad técnica. Caso de estudio}

\subsection{1.- Antecedentes}

El análisis de viabilidad técnica se realiza en base a un caso práctico en el Municipio de Haría, Las Palmas, concretamente en el pueblo de Tabayesco. En esta localidad ha salido recientemente a licitación pública, el proyecto denominado "Renovación del alumbrado público del pueblo de Tabayesco", en un proyecto gestionado por su Cabildo Insular. El proyecto abarca la canalización subterránea del suministro de electricidad, así como la instalación de nuevas luminarias eficientes de tipo LED.

En un análisis de viabilidad técnica, se lleva a cabo el dimensionado de una instalación de iluminación LED alimentada con energía solar fotovoltaica que dé servicio a esta localidad objeto de estudio, cumpliendo con los requisitos lumínicos fijados en el concurso público empleado como referencia.

\subsection{2.- Descripción del problema}

Las administraciones públicas locales, en virtud de las carencias detectadas en una auditoría energética realizada, se han visto obligadas a elaborar un plan de renovación del alumbrado público municipal, donde se propone la adecuación a la normativa el suministro eléctrico que da servicio al alumbrado público.

Entre estas medidas, se contempla la canalización subterránea del tendido eléctrico, pues como se comprueba en la figura 1, es muy habitual la conducción eléctrica por fachadas.

Figura 1. Identificación del problema en estudio
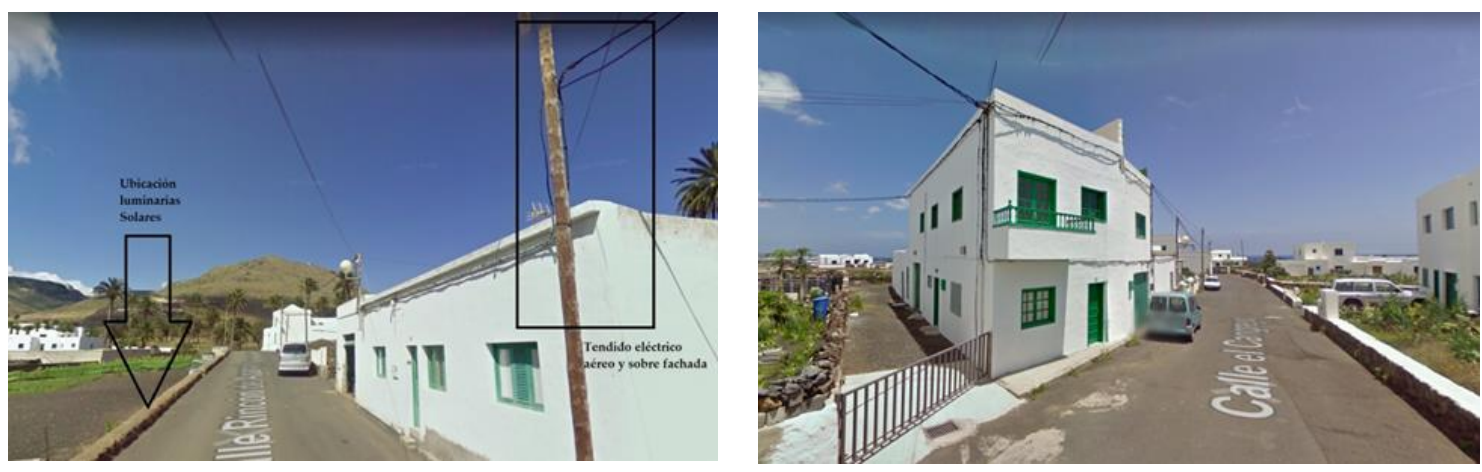

Fuente: Google Maps Visto en: febrero 2018

WPS RI-SHUR, nำ-8, 2018, vol.1, ISSN: 2387-1768 


\section{WPSReview International on Sustainable \\ Housing and Urban Renewal (RI-SHUR)}

\subsection{3.- Emplazamiento}

La instalación prevista estará formada, en una primera fase, por una actuación sobre las calles principales del núcleo urbano con un total de 76 puntos de luz y una segunda fase que comprende la ejecución de las obras e instalaciones en las vías que carecen actualmente de asfaltado, que suma un total de 23 puntos de luz.

Figura 2. Emplazamiento. Pueblo de Tabayesco, Haría, Las Palmas
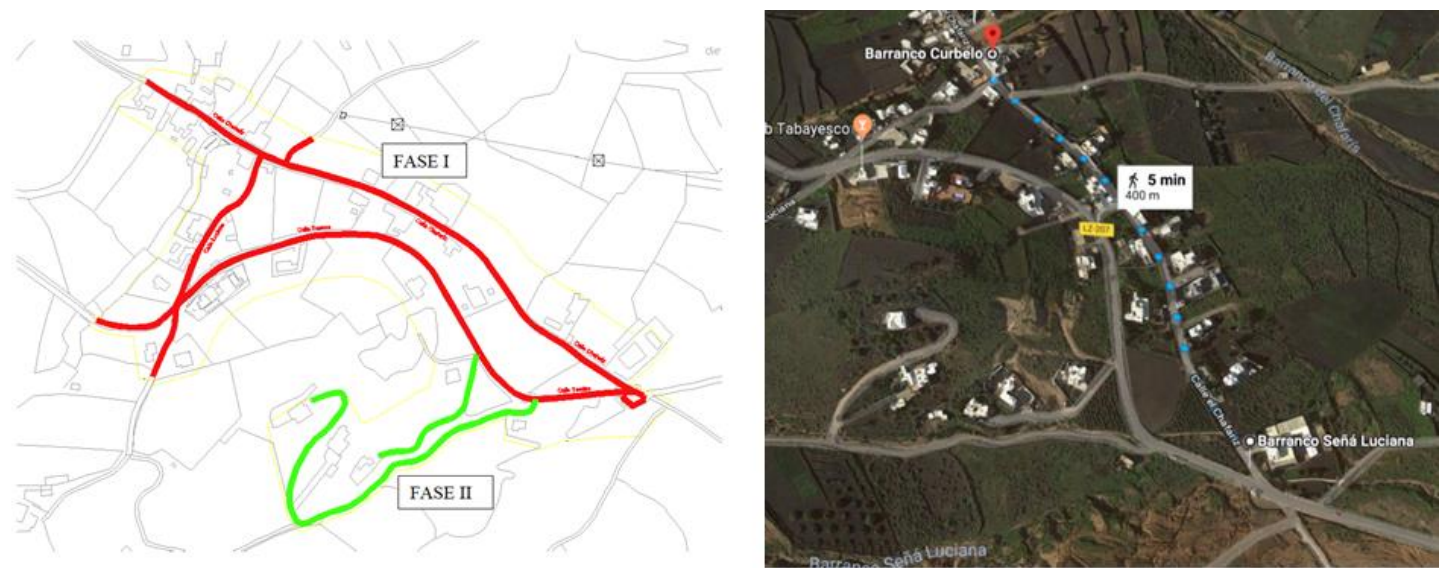

Fuente: Google Maps. Visto en: febrero 2018

\subsection{4.- Dimensionado de las luminarias}

La zona objeto de actuación, queda clasificada como Zona E1, definida como áreas con entornos o paisajes oscuros, destinadas a observatorios astronómicos de categoría internacional, parques nacionales, espacios de interés natural, áreas de protección especial (red natura, zonas de protección de aves, ...) donde las carreteras están sin iluminar. Esto implica unas condiciones operativas específicas, como una temperatura de color de las luminarias en valores cálidos $(3.000 \mathrm{~K})$ o un flujo hacia el hemisferio superior (FHSINST) inferior o igual al 1\% del total emitido. En base a sus condiciones específicas se ha clasificado la vía como ME4b.

Con los requisitos mencionados y conociendo los parámetros geométricos de la calzada, se dimensiona la potencia de las luminarias con el software gratuito DIALux. En el proyecto de referencia se establecen dos tipos de calles, los resultados se muestran resumidos en la tabla 3. 


\section{WPSReview International on Sustainable \\ Housing and Urban Renewal (RI-SHUR)}

4.1.5.- Dimensionado del panel fotovoltaico, sistema de acumulación y sistema de regulación

En la tabla 4 aparecen resumidas las ecuaciones que permiten el dimensionado de los componentes restantes de la instalación, como son el panel fotovoltaico, el sistema de almacenamiento y el sistema de regulación.

\subsection{6.- Componentes de la instalación propuesta}

En la tabla 5 se pueden visualizar los componentes que conforman la instalación solar LED dimensionada para la localidad objeto de estudio.

\subsection{7.- Simulación de la instalación propuesta}

En este apartado se resumen los resultados de la simulación luminotécnica, el modelado 3D y el renderizado final. Este modelo, pese a tener un enfoque aparentemente estético, se realiza para enriquecer el estudio de viabilidad social, pues permite a los encuestados tener una proyección tridimensional de la instalación propuesta.

\section{2.- Análisis de viabilidad económica}

En primer lugar, se comparan los resultados obtenidos en un análisis de licitaciones públicas en España enfocadas a la renovación del alumbrado público entre 2016 y 2018, y especialmente aquellas que proponen luminarias alimentadas con energía solar. Dicho estudio puede visualizarse en la tabla 6 . Se concluye que, el precio medio por luminaria solar LED para licitaciones públicas en el territorio español es de $1.875,24 €$, con mástil, cimentación, instalación, gastos generales, beneficio industrial e impuestos incluidos

En segundo lugar, se toma como referencia la licitación pública mencionada en el análisis de la viabilidad técnica, donde se describe la renovación del alumbrado del pueblo de Tabayesco, Las Palmas. Esta instalación implica la canalización subterránea del suministro eléctrico, así como la instalación de nuevas luminarias LED. El valor estimado del contrato se fija en $326.960,38 €$, por un total de 99 luminarias LED. Por tanto, se puede concluir que el precio medio de cada luminaria LED para esta licitación es de 3.302,63 €/ud.

Por último, tras un estudio de viabilidad técnica en el que se dimensiona una instalación de iluminación alimentada con energía solar fotovoltaica, que cumpla con los mismos requisitos que la instalación de referencia, se determina un presupuesto de $183.861,70 €$ por un total de 99 luminarias solares LED. Por tanto, se puede concluir que el precio medio de cada luminaria solar para una licitación de estas características es de $1.857,19 € /$ ud.

WPS RI-SHUR, nำ7-8, 2018, vol.1, ISSN: 2387-1768 


\section{WPSReview International on Sustainable}

\section{Housing and Urban Renewal (RI-SHUR)}

Tabla 3. Datos geométricos de las vías de estudio

\begin{tabular}{|c|c|c|}
\hline Tipo de Vía & 1 & 2 \\
\hline Clasificación de la vía & ME4b & ME4b \\
\hline Número de carriles & 1 & 1 \\
\hline Ancho de calzada (m) & 7 & 6 \\
\hline Interdistancia (m) & 18 & 21,5 \\
\hline Altura del punto de luz (m) & 6 & 6 \\
\hline Disposición & Unilateral & Unilateral \\
\hline Potencia Luminarias (Referencia) & 58,1 & 58,1 \\
\hline Potencia Luminarias (Solar LED) & 40 & 40 \\
\hline Luminaria (Proyecto de referencia) & 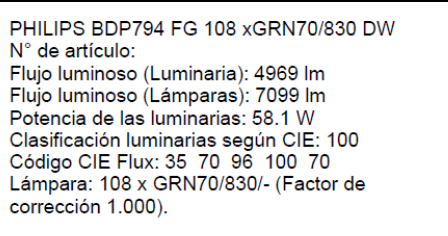 & \\
\hline $\begin{array}{l}\text { Luminarias (Propuesta iluminación } \\
\text { Solar LED) }\end{array}$ & 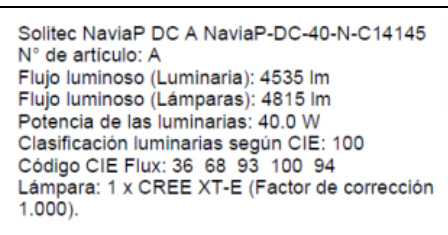 & \\
\hline
\end{tabular}

Fuente: elaboración propia

WPS RI-SHUR, no7-8, 2018, vol.1, ISSN: 2387-1768 
WPSReview International on Sustainable

Housing and Urban Renewal (RI-SHUR)

Tabla 4. Ecuaciones para el dimensionado de instalaciones de iluminación LED alimentadas con energía solar fotovoltaica

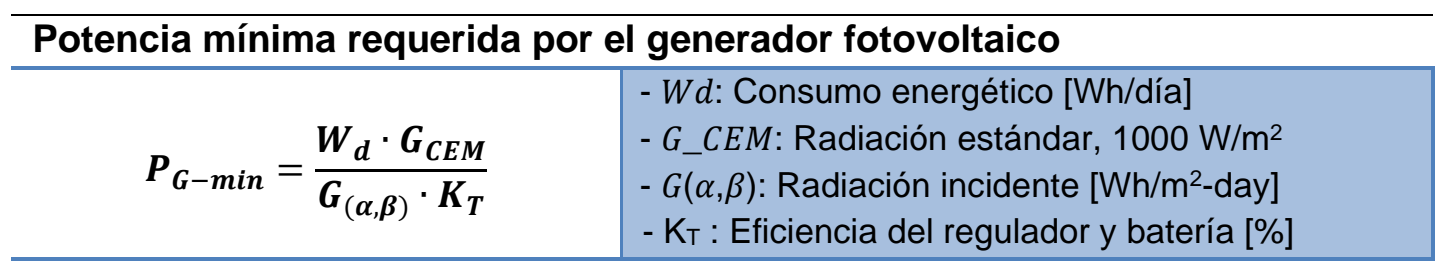

\section{Sistema de acumulación}

\begin{tabular}{|c|c|}
\hline$C_{N}=\frac{Q_{d} \cdot A}{P D_{M A X} \cdot \eta_{C o n}}$ & $\begin{array}{l}\text { - Qd: Capacidad nominal diaria [Ah/día] } \\
\text { - A: Días de autonomía } \\
\text { - PDmAx: Profundidad de descarga máxima } \\
\text { - } \eta \text { _con: Eficiencia del regulador y batería } \\
\text { - Un: Tensión nominal del generador fotovoltaico } \\
\text { [V] }\end{array}$ \\
\hline \multicolumn{2}{|l|}{ Sistema de regulación } \\
\hline $\begin{array}{c}U_{O C(\text { Tmin })}=U_{O C}+\beta \\
\cdot\left(T_{\min }-25\right) \\
I_{R}=1,25 \cdot I_{G, S C}\end{array}$ & $\begin{array}{l}\text { - Uoc (TMIN): Tensión nominal del generador } \\
\text { fotovoltaico [V] } \\
\text { - Tmin: Temperatura mínima histórica }\left({ }^{\circ} \mathrm{C}\right) \\
\text { - Uoc: Tensión de circuito abierto }[\mathrm{V}] \\
\text { - IG,SC : Intensidad de cortocircuito del generador } \\
\text { [A] }\end{array}$ \\
\hline
\end{tabular}

Fuente: Elaboración propia 
WPSReview International on Sustainable

Housing and Urban Renewal (RI-SHUR)

Tabla 5. Componentes de la instalación propuesta

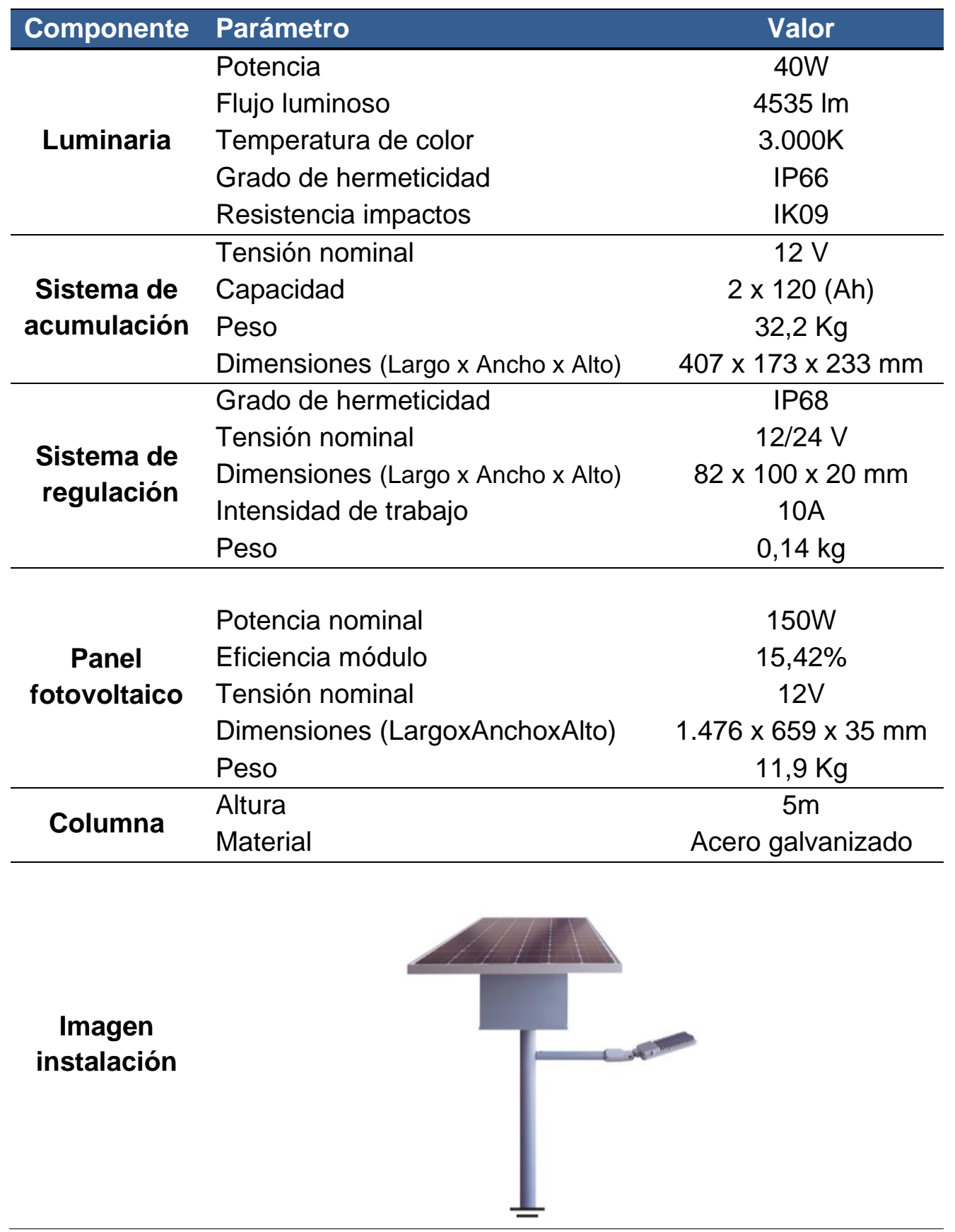

Fuente: Elaboración propia

WPS RI-SHUR, nำ-8, 2018, vol.1, ISSN: 2387-1768 


\section{WPSReview International on Sustainable}

\section{Housing and Urban Renewal (RI-SHUR)}

Tabla 6. Licitaciones públicas en España entre 2016 y 2017

\begin{tabular}{|c|c|c|c|c|c|}
\hline $\begin{array}{l}\text { Lugar y fecha de } \\
\text { publicación }\end{array}$ & Sistema propuesto & $\begin{array}{c}\text { NN2 de } \\
\text { luminarias }\end{array}$ & $\begin{array}{c}\text { Potencia } \\
\text { Luminarias }(W)\end{array}$ & $\begin{array}{c}\text { Presupuesto adjudicación / } \\
\text { Precio medio licitación }\end{array}$ & $\begin{array}{l}\text { Presupuesto adjudicación / } \\
\text { Precio medio adju dicación }\end{array}$ \\
\hline $\begin{array}{l}\text { Lloseta, Islas Baleares } \\
\text { (Septiembre 2016) }\end{array}$ & \begin{tabular}{|c|} 
Luminaria vial LED \\
Batería, regulador y panel \\
PV integrados en cuerpo \\
luminaria
\end{tabular} & 39 & 40 & $49.957,33 € / 1.280,96 €$ & - \\
\hline $\begin{array}{c}\text { Calpe, Comunidad } \\
\text { Valenciana (Agosto } \\
\text { 2017) }\end{array}$ & $\begin{array}{c}\text { Luminaria vial LED } \\
\text { Batería y regulador } \\
\text { integrados en el cuerpo } \\
\text { de la luminaria } \\
\text { Panel PV independiente }\end{array}$ & 102 & 30 & $130.000 € / 1.274,51 €$ & - \\
\hline $\begin{array}{l}\text { Pamplona, Navarra } \\
\text { (Noviembre 2016) }\end{array}$ & \begin{tabular}{|c|} 
Luminaria vial LED \\
Batería, regulador y panel \\
PV no integrados en \\
cuerpo luminaria
\end{tabular} & 31 & 30 & $95.397,58 € / 3.077,34 €$ & - \\
\hline $\begin{array}{l}\text { Málaga, Andalucía } \\
\text { (Diciembre 2017) }\end{array}$ & \begin{tabular}{|c|} 
Luminaria vial LED \\
Batería, regulador y panel \\
PV no integrados en \\
cuerpo luminaria \\
\end{tabular} & 72 & 30 & $177.881,83 € / 2.470,58 €$ & - \\
\hline $\begin{array}{l}\text { La Antigua, Las } \\
\text { Palmas (Noviembre } \\
\text { 2016) }\end{array}$ & \begin{tabular}{|c|} 
Luminaria villa LED \\
Batería, regulador y panel \\
PV no integrados en \\
cuerpo luminaria \\
\end{tabular} & 75 & 42 & $118.645,61 € / 1.581,94 €$ & $11.1815 € / 1.490,87 €$ \\
\hline $\begin{array}{l}\text { Málaga, Andalucía } \\
\text { (Septiembre 2016) }\end{array}$ & \begin{tabular}{|c|} 
Luminaria vial LED \\
Batería, regulador y panel \\
PV no integrados en \\
cuerpo luminaria
\end{tabular} & 38 & 30 & $91.857,15 € / 2.417,29 €$ & $86.345,72 € / 2.272,26 €$ \\
\hline $\begin{array}{c}\text { Eivissa, Islas Baleares } \\
\text { (Abril 2017) }\end{array}$ & \begin{tabular}{|} 
Luminaria vial LED \\
B atería, regulador y panel \\
PV no integrados en \\
cuerpo luminaria
\end{tabular} & 67 & 30 & $170.903,21 € / 2.550,79 €$ & $159.921,43 € / 2.386,89 €$ \\
\hline
\end{tabular}

Fuente: Elaboración propia 


\section{WPSReview International on Sustainable \\ Housing and Urban Renewal (RI-SHUR)}

Tabla 7. Resultados del estudio de viabilidad económica

\begin{tabular}{|c|c|c|c|c|c|c|}
\hline & \multicolumn{3}{|c|}{ Resultados energéticos } & \multicolumn{3}{|c|}{ Resultados económicos } \\
\hline & Potencia luminaria & $\begin{array}{c}\text { Energía } \\
\text { consumida }\end{array}$ & $\begin{array}{c}\text { Potencia } \\
\text { instlada }\end{array}$ & \begin{tabular}{|c|}
$\begin{array}{c}\text { Presupuesto total } \\
\text { instalación }\end{array}$ \\
\end{tabular} & $\begin{array}{c}\text { Precio total } \\
\text { por luminaria }\end{array}$ & \begin{tabular}{|c|} 
Disminución \\
presupuesto total \\
\end{tabular} \\
\hline $\begin{array}{l}\text { Instalación de } \\
\text { referencia }\end{array}$ & $58,1 \mathrm{~W}$ & 6995,78 kWh-año & $5,75 \mathrm{~kW}$ & $326.960,38 €$ & $3.302,63 €$ & $0,00 \%$ \\
\hline $\begin{array}{l}\text { Instalación solar } \\
\text { LED (1). Estudio } \\
\text { concursos públicos }\end{array}$ & $32,5 \mathrm{~W}$ & 0 kWh-año & $0 \mathrm{~kW}$ & $185.648,76 €$ & $1.875,24 €$ & $43,22 \%$ \\
\hline $\begin{array}{l}\text { Instalación solar } \\
\text { LED (2). Estudio } \\
\text { viablidad técnica }\end{array}$ & $40 \mathrm{~W}$ & 0 kWh-año & $0 \mathrm{~kW}$ & $183.861,70 €$ & $1.857,19 €$ & $43,77 \%$ \\
\hline Anotaciones & $\begin{array}{l}\text { Se reduce la potencia por } \\
\text { luminaria un } 31,15 \% \text { en la } \\
\text { instalación solar LED (2) } \\
\text { con respecto a la } \\
\text { instalación de referencia }\end{array}$ & \multicolumn{2}{|c|}{$\begin{array}{l}\text { Las instalaciones solares son } \\
\text { autónomas por tanto el } \\
\text { consumo de energía de la red es } \\
\text { nulo y por tanto la potencia } \\
\text { instalada es } 0 \mathrm{~kW} \text {. }\end{array}$} & \multicolumn{3}{|c|}{$\begin{array}{c}\text { Presupuesto total de la instalacion. En la instalación } \\
\text { de referencia: } 67 \text { puntos de luz con canalización en } \\
\text { calzada asfaltada y } 33 \text { en camino de tierra. Todas las } \\
\text { instalaciones incluyen gastos generales, beneficio } \\
\text { industrial e impuestos }\end{array}$} \\
\hline
\end{tabular}

\section{3.- Análisis de viabilidad social. Estudio de la percepción ciudadana}

Neeraj Kumar Singh et al (Singh NK, 2017) realizaron una encuesta compuesta por 14 preguntas acerca de la opinión pública en referencia al uso de energía solar fotovoltaica en diferentes regiones de la India. Los resultados incluyen opiniones muy diversas, destacando que la mayoría de los encuestados no tiene una idea clara de lo que es la energía solar fotovoltaica y sus aplicaciones.

Con el objetivo de obtener la opinión pública, en nuestro caso, de la sociedad española sobre esta tecnológica y la viabilidad de implantarla en nuestras ciudades, se ha realizado estudio estadístico basado en una encuesta en el que se realizan preguntas sobre este tema a 349 sujetos anónimos. La encuesta se ha realizado mediante Google Forms y puede consultarse o realizarse a través del siguiente enlace público: https://goo.gl/forms/Bva0fUczehwi2u0z2

Es de reseñar que casi el $60 \%$ de los encuestados corresponde a un sector de la población con una edad inferior a los 25 años. Una posible explicación se atribuye al hecho de realizarse online, donde existe una mayor difusión entre el público joven. 


\section{WPSReview International on Sustainable}

\section{Housing and Urban Renewal (RI-SHUR)}

Figura 3. Resumen de resultados de la encuesta de valoración de la aceptación social del alumbrado solar fotovoltaico en entornos urbanos

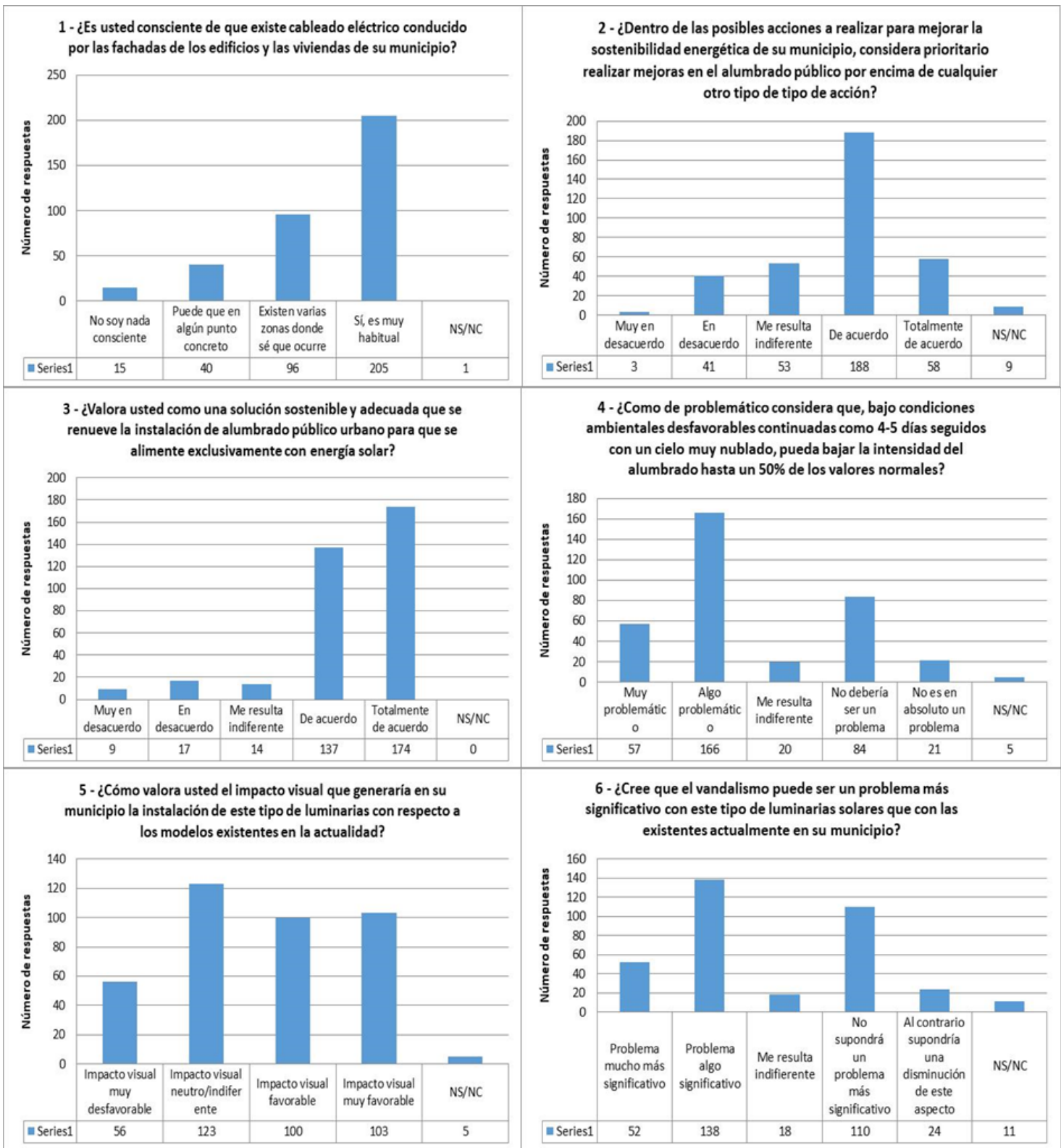

Fuente: Elaboración propia

Los principales resultados del estudio se presentan en la figura 3. De estos se pueden extraer las siguientes conclusiones:

- La gran mayoría de la población es consciente de que existe cableado eléctrico conducido por las fachadas de los edificios. Tan solo el $4 \%$ de los encuestados afirma no ser consciente.

WPS RI-SHUR, no7-8, 2018, vol.1, ISSN: 2387-1768 


\title{
WPSReview International on Sustainable
}

\author{
Housing and Urban Renewal (RI-SHUR)
}

- Casi el $60 \%$ de los encuestados están de acuerdo o totalmente de acuerdo en considerar prioritario realizar mejoras en el alumbrado público por encima de cualquier otro tipo de acción para mejorar la sostenibilidad energética de su municipio.

- El $89 \%$ considera una solución sostenible y adecuada que se renueve la instalación de iluminación urbana para que se alimente exclusivamente con energía solar fotovoltaica.

- La percepción ante el problema de que, bajo condiciones ambientales desfavorables continuadas como 4-5 días seguidos con un cielo muy nublado, pueda bajar la intensidad del alumbrado hasta un $50 \%$ de los valores normales para garantizar el servicio. El $47 \%$ de los encuestados lo considera algo problemático y el $16 \%$ muy problemático.

- En referencia a la percepción visual de la tecnología propuesta frente a la convencional, un $32 \%$ lo considera neutro/indiferente, por otra parte, más del $50 \%$ encuentra favorable el cambio, siendo muy favorable o favorable con un $27 \%$ y $26 \%$ respectivamente.

- Sobre el posible incremento del vandalismo sobre este equipamiento urbano, más de la mitad de los encuestados considera que el problema del vandalismo puede agravarse, siendo considerado un problema mucho más significativo 0 algo significativo con un $15 \%$ y $39 \%$ respectivamente.

\section{5.- Ejemplos}

\section{1.- Cuimba, Angola}

En el contexto de esta investigación, se ha participado en un proyecto de alumbrado público compuesto por 1.365 luminarias autónomas LED de 50W en la ciudad de Cuimba (Angola), como se ilustra en la figura 4. El presupuesto de instalar este tipo de equipos de iluminación se redujo en más del $35 \%$ en comparación con la opción analizada para renovar y canalizar la red eléctrica existente para un sistema de alumbrado alimentado en red.

\section{Figura 4. Cimentación, instalación y puesta en marcha de la instalación}
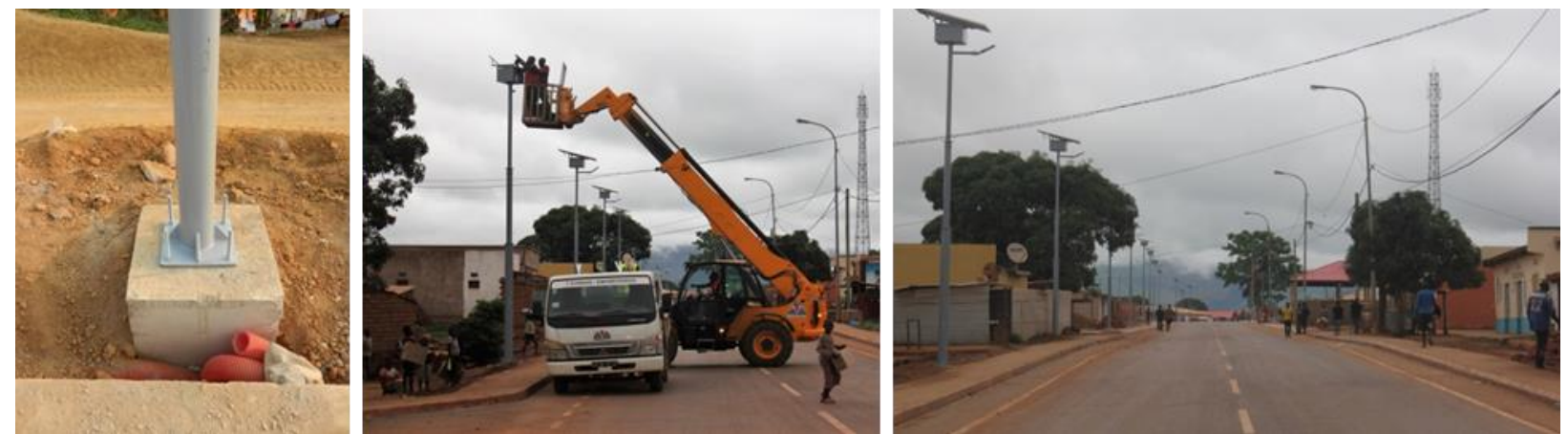

WPS RI-SHUR, no7-8, 2018, vol.1, ISSN: 2387-1768 


\section{WPSReview International on Sustainable}

Housing and Urban Renewal (RI-SHUR)

\section{2.- Brisbane, Australia}

En un proyecto subvencionado por la Universidad de Queensland (Brisbane), se ha determinado como viable para este tipo de localizaciones, la opción de reemplazar el sistema de alumbrado de la ciudad con luminarias solares LED (Jiang X., 2016).

\section{3.- Jordania}

En un estudio de viabilidad económica desarrollado por Lina Al-Kurdi et al (2015), que investiga la posibilidad de emplear sistemas de iluminación solar LED en el alumbrado público de Jordania, se determinó que se obtenían ahorros del $50 \%$ con respecto a la instalación actual existente. En cuanto a los periodos de amortización, se obtuvieron los siguientes resultados:

- Periodo de amortización de 7,20 años para instalaciones de iluminación LED conectadas a la red de corriente alterna.

- Periodo de amortización de 3,15 años para instalaciones de iluminación LED alimentadas con energía solar fotovoltaica.

\section{4.- Indonesia}

Ika Shinta Mardikaningsih et al (Mardikaningsih IS, 2016), realizan un estudio de viabilidad técnico-económica empleando métodos como el VAN o la relación costebeneficio con una proyección a 25 años. Determinan que la inversión empleando luminarias solares para alumbrado urbano como factible, siendo un $97 \%$ más rentable que el actual empleo alimentación en red con un componente principal de energía basado en combustibles fósiles.

\section{5.- Islas Canarias, España}

De un estudio propio de la viabilidad técnica-económica de instalaciones de iluminación solar LED en el término municipal de Haría, Las Palmas, se concluye que el empleo de esta tecnología supone un ahorro en un presupuesto de ejecución material de un $43,77 \%$ con respecto a la canalización subterránea e instalación de nuevas luminarias LED conectadas a la red de corriente alterna, necesaria para la adecuación a la normativa de las instalaciones eléctricas existentes aéreas y en superficie por las fachadas de las viviendas. Esta valoración del ahorro se ha realizado usando la 26a Edición de la Base de Precios de Construcción en Canarias y la $5^{\text {a }}$ edición de la Base de Precios de Obras Viales en Canarias. 


\section{WPSReview International on Sustainable}

Housing and Urban Renewal (RI-SHUR)

Figura 5. Instalación de iluminación solar LED propuesta en el estudio de viabilidad técnico-económico,
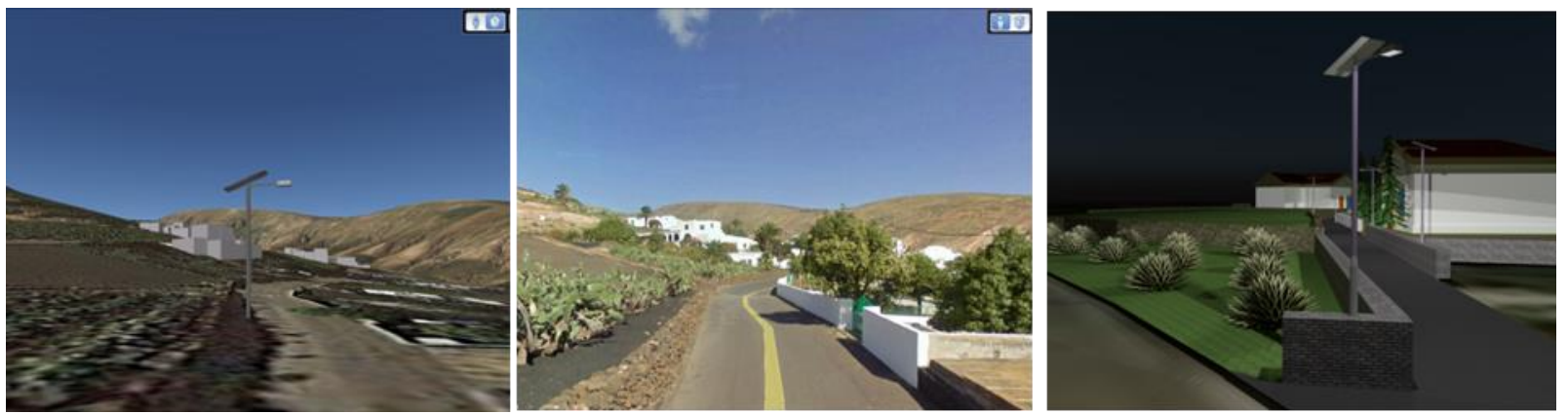

Haría, Las Palmas

\section{6.- Conclusiones y línea de trabajo futura}

Se concluye que las instalaciones de alumbrado público urbano fotovoltaico resultan técnica, económica y socialmente viables en virtud de los resultados obtenidos.

El análisis de viabilidad técnica, realizado en el marco de un caso práctico, permite visualizar la instalación y comprobar que la tecnología propuesta cumple de manera contundente con los requisitos normativos. Este estudio se ve contrastado con el análisis de los concursos públicos en España, donde se comprueba como las luminarias solares LED comienzan a adquirir protagonismo en los últimos años.

Por su parte, el estudio de viabilidad económica proporciona los resultados más significativos, determinando que resulta un $44 \%$ más viable la opción de realizar una instalación de iluminación LED PV con respecto a una instalación de iluminación LED conectada a la red de corriente alterna y adaptada a la normativa vigente mediante la canalización subterránea.

Por último, del estudio de viabilidad social se concluye que la mayoría de la población es consciente del problema de la conducción aérea y sobre fachadas y, a su vez, el $89 \%$ considera una solución sostenible y adecuada que se renueve la instalación de iluminación urbana para que se alimente exclusivamente con energía solar fotovoltaica. Por su parte, la mayoría de los encuestados valora como problemático el hecho de que, bajo condiciones ambientales desfavorables continuadas, pueda bajar la intensidad del alumbrado para garantizar el servicio y más de la mitad considera que el problema del vandalismo puede agravarse.

Las exigencias medioambientales, la creciente apuesta internacional en el empleo de fuentes de energías renovables y la probada viabilidad de la tecnología de iluminación LED PV da lugar a que se hagan pronósticos alentadores a favor de ésta tecnología, esperando su implantación progresiva en zonas con un buen recurso solar. 


\section{WPSReview International on Sustainable \\ Housing and Urban Renewal (RI-SHUR)}

\section{7.-. Referencias bibliográficas}

Alliance for Rural Electrification (ARE). Rural electrification with renewable energy: Technologies, quality standards and business models, www.ruralelec.org

Battaglini A, Komendantova N, Brtnik P y Patt A. Perception of barriers for expansion of electricity grids in the European Union. Energy Policy. 2012.47; pp. 254-259.

Breyer, C, Bogdanov, D, Gulagi, A, Aghahosseini, A, Barbosa, LS, Koskinen, O y Farfan, J. (2017). On the role of solar photovoltaics in global energy transition scenarios. Progress in Photovoltaics: Research and Applications, 25(8), 727-745.

Carrasco-Moreno LM. Characterisation of the operation \& maintenanse phase in pv rural electrification programmes. Tésis doctoral, Universidad Politécnica de Madrid. 2015.

Gago A, Fraile J. Iluminación con tecnología LED. Ediciones Paraninfo S. A., 2012.

Gils, HC, Scholz, Y, Pregger, T, de Tena, DL y Heide, D. (2017). Integrated modelling of variable renewable energy-based power supply in Europe. Energy, 123, 173-188.

Gutierrez-Escolar A, Castillo-Martinez A, Gomez-Pulido JM, Gutierrez-Martinez JM, Stapic Z, Medina-Merodio JA. A study to improve the quality of street lighting in Spain. Energies, 8(2), 976-994. 2015

Hyder F, Sudhakar K, y Mamat R. Solar PV tree design: A review. Renewable and Sustainable Energy Reviews. 2018; 82, pp. 1079-1096.

Jiang X. Innovation to Brisbane City Council Street Lighting System with Solar Powered LED. University of Queensland. Australasian Universities Power Engineering Conference- AUPEC2016

Lau SP, Weddell AS, Merrett GV, White NM. Energy-neutral solar-powered street lighting with predictive and adaptive behaviour. In Proceedings of the 2nd International Workshop on Energy Neutral Sensing Systems (pp. 13-18). ACM. 2014

Mardikaningsih IK, Sutopo W, Hisjam M, Zakaria R. Techno-economic Feasibility Analysis of a Public Street Light with Solar Cell Power. Proceedings of the International MultiConference of Engineers and Computer Scientists 2016 Vol II, IMECS 2016, March 16 - 18, 2016, Hong Kong.

Montoya, FG, Peña-García, A, Juaidi, A y Manzano-Agugliaro, F. (2017). Indoor lighting techniques: An overview of evolution and new trends for energy saving. Energy and Buildings, 140, 50-60.

Nowak S. Trends in photovoltaic applications, Report IEA-PVPS T1-27:2015, IEA International Energy Agency, 2015. 
WPSReview International on Sustainable

Housing and Urban Renewal (RI-SHUR)

Singh NK, Gupta R, Salimath GF, Badge SS. Public opinion on solar photovoltaic energy utilization-A survey based study. In Proceedings of International Conference on. Smart and Sustainable Initiatives for Energy within Environmental Constraints. 2017, Vol. 1, pp. 28-34, Malaysia.

Ramchandra P. y Diouf B. Solar lighting. Springer Science \& Business Media, 2011. 